\title{
Superior Vena Cava Cancerous Thrombus Due to Squamous Cell Tongue Cancer: Virchow's Triad at Its Worst Version
}

\author{
John Papanikolaou ${ }^{1,2}$ Triantafyllia Koukoubani ${ }^{3}$ Epaminondas Zakynthinos ${ }^{2}$ \\ ${ }^{1}$ Department of Cardiology, General Hospital of Trikala, \\ Thessaly, Greece \\ 2 Department of Critical Care, University Hospital of Larissa, \\ Thessaly, Greece \\ ${ }^{3}$ Department of Critical Care, General Hospital of Trikala, \\ Thessaly, Greece \\ Thromb Haemost 2019;119:512-514. \\ Address for correspondence John Papanikolaou, MD, PhD, Biopolis, \\ 41110 Larissa, Greece (e-mail: y_papanikolaou@hotmail.com).
}

\begin{abstract}
Keywords

- superior vena cava syndrome

- cancerous thrombus

- squamous cell tongue cancer

- pericardial effusion

Superior vena cava (SVC) cancerous thrombosis is extremely sparse, especially in the setting of extrathoracic tumours. Herein, we present the case of a patient with a squamous cell carcinoma of the tongue, who presented with SVC syndrome possibly secondary to symptomatic metastatic pericardial effusion. In this unique patient, the disastrous concurrence of all the elements of Virchow's triad within the confined anatomical space of SVC may have precipitated extensive vessel thrombosis with catastrophic consequences. To our knowledge, there has been no previous report about presence of SVC cancerous thrombosis in squamous cell tongue cancer. In this respect, our report may provide an unusual mechanism of tongue cancer expansion, which clinicians should be familiar with. In addition, it may highlight the clinical importance of SVC thrombosis on patients' clinical outcome, as well as the role of transoesophageal echocardiography in early detection of occult thrombi in the sub-set of patients with SVC syndrome.
\end{abstract}

A 48-year-old man with a 6-month history of poorly differentiated squamous cell carcinoma of the tongue presented with clinical and echocardiographic signs of cardiac tamponade. He also demonstrated signs compatible with superior vena cava (SVC) syndrome (plethoric face and oedematous arms bilaterally). Emergent pericardiocentesis yielded 1,200 $\mathrm{mL}$ of haemorrhagic fluid, resulting in temporary clinical improvement.

Interestingly, post-drainage transthoracic sub-costal (-Fig. 1A) and transoesophageal echocardiographic (TEE) aortic-valve short-axis ( - Video 1 ) views disclosed a highly mobile ball-like thrombus within the right atrium, initially considered to be 'en route' to pulmonary circulation. However, TEE bi-caval view revealed that the thrombus was actually extremely elongated, mainly located within SVC; while its lower edge demonstrated a brisk, whip-like motion within

the right atrium ( $\mathbf{- F i g}$. 1B, - Video 2), vascular ultrasonography of the neck (- Figs. 1C and D) illustrated that SVC thrombus was extended up to the internal jugular veins (IJVs), which were both invaded by the surrounding metastatic lymph nodes and thrombosed. Combined cardiovascular ultrasonography was compatible with the presence of cancerous thrombus within SVC. ${ }^{1}$ Computed tomography pulmonary angiography confirmed ultrasound findings, illustrating contrast deficits (open arrows) within the right atrium ( - Fig. 2A), SVC ( - Fig. 2B), both brachiocephalic veins ( - Fig. 2C) and IJVs (-Fig. 2D); it also disclosed sub-segmental thrombi (arrow heads) in the lower lung lobes compatible with on-going pulmonary embolism ( - Figs. $2 \mathbf{A}$ and $\mathbf{B}$ ). The patient received anticoagulants, however, his clinical course was complicated by major pulmonary embolism and sepsis, and he died before resorting to surgery. @ 2019 Georg Thieme Verlag KG
Stuttgart · New York
DOI https://doi.org/

10.1055/s-0039-1677712. ISSN 0340-6245. 

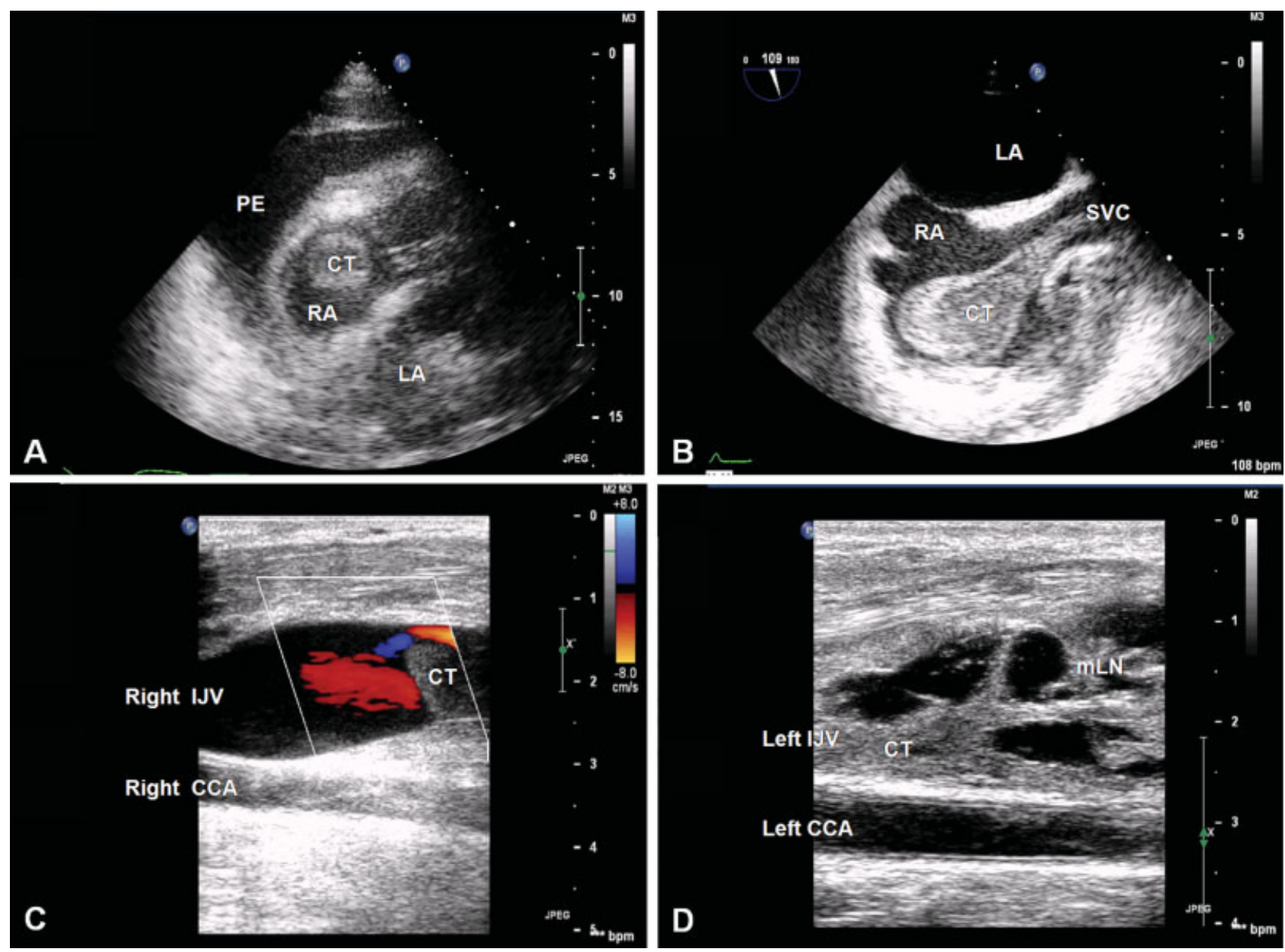

Fig. 1 Superior vena cava cancerous thrombus in transthoracic sub-costal view (A), transesophageal bi-caval view (B), right (C) and left (D) neck vascular ultrasonography (see also text).

\section{Video 1}

Cancerous thrombus in transesophageal short-axis view at the level of aortic valve (see also text). CT, cancerous thrombus; CCA, common carotid artery; IJV, internal jugular vein; LA, left atrium; $\mathrm{mLN}$, metastatic lymph node; PE, pericardial effusion; RA, right atrium; SVC, superior vena cava. Online content including video sequences viewable at: www.thiemeconnect.de/ products/ejournals/html/10.1055/s-0039-1677712.

\section{Video 2}

Superior vena cava cancerous thrombus in transesophageal bi-caval view (see also text). CT, cancerous thrombus; CCA, common carotid artery; IJV, internal jugular vein; LA, left atrium; mLN, metastatic lymph node; PE, pericardial effusion; RA, right atrium; SVC, superior vena cava. Online content including video sequences viewable at: www.thiemeconnect.de/ products/ejournals/html/10.1055/s-0039-1677712.
To our knowledge, there has been no previous report about the presence of SVC cancerous thrombosis in squamous cell tongue cancer. ${ }^{2}$ Notably, all of the three Virchow's components ${ }^{3}$ were present in our patient's SVC system, including metastatic ${ }^{4}$ pericardial effusion-induced blood stasis, vascular tumour invasion and malignancy-associated hypercoagulable state. ${ }^{5}$ This catastrophic triad might have played a pivotal role in precipitating fatal thrombogenesis in our rare patient. We also hypothesize that abrupt decrease of intrapericardial pressure after pericardiocentesis may have 'opened the bag of Aeolus', resulting in thrombus prolapse within the right heart and fatal pulmonary embolism. Certainly, case reports are of less clinical value in advancing scientific knowledge and reviewing current therapeutic algorithms in rare diseases. However, our rare report may provide an unusual mechanism of tongue cancer expansion, which clinicians should be familiar with. In addition, it may highlight the clinical importance of TEE and vascular ultrasound in early detection of occult thrombi in patients with SVC syndrome. 

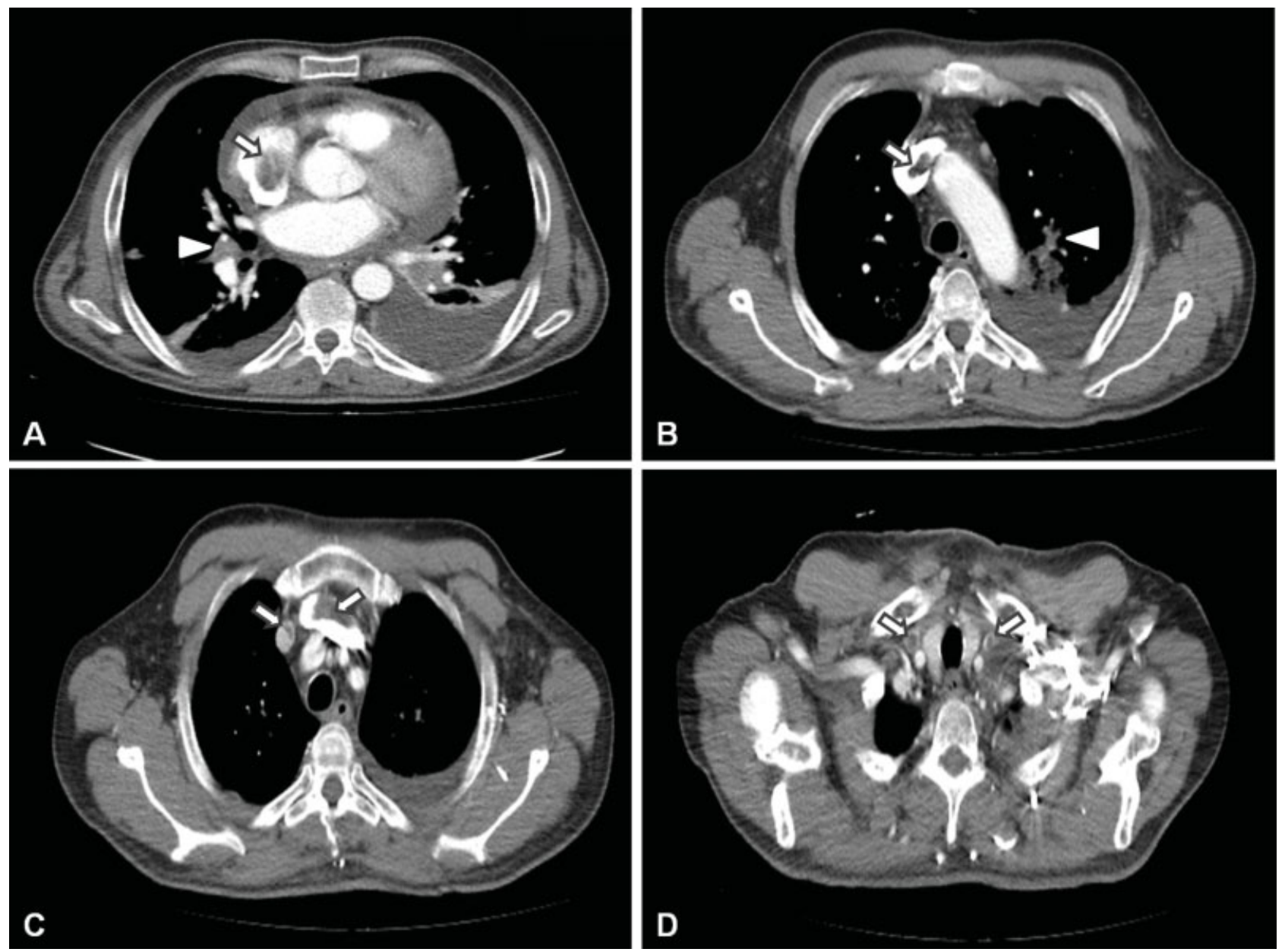

Fig. 2 Computed tomography pulmonary angiography illustrating superior vena cava (SVC) cancerous thrombus within right atrium (A), SVC per se (B), brachiocephalic (C) and internal jugular veins (D) (see also text).

Authors' Contributions

J.P. and E.Z. conceived the study. J.P., T.F. and E.Z. participated in the acquisition and interpretation of data, drafted and critically revised the manuscript for important intellectual content. All authors read and approved the final manuscript.

\section{Funding}

None.

Conflict of Interest

None declared.

\section{References}

1 Kraft C, Schuettfort G, Weil Y, et al. Thrombosis of the inferior vena cava and malignant disease. Thromb Res 2014;134(03):668-673

2 Wilson LD, Detterbeck FC, Yahalom J. Clinical practice. Superior vena cava syndrome with malignant causes. N Engl J Med 2007; 356(18):1862-1869

3 Bagot CN, Arya R. Virchow and his triad: a question of attribution. Br J Haematol 2008;143(02):180-190

4 Rivkin A, Meara JG, Li KK, Potter C, Wenokur R. Squamous cell metastasis from the tongue to the myocardium presenting as pericardial effusion. Otolaryngol Head Neck Surg 1999;120(04):593-595

5 Kimura T, Chino M, Ogasawara N, Nakano T, Izumi S, Takeuchi H. Trousseau's syndrome with brachiocephalic vein thrombosis in a patient with uterine carcinosarcoma. A case report. Angiology 1999;50(06):515-518 